\title{
Route selection strategy for Hybrid vehicles based on Energy Management and real time drive cycles
}

\author{
Hector Garica Pimentel, Oxford Brookes University \\ Stephen Samuel, Oxford Brookes University
}

\begin{abstract}
Air pollution levels in an urban environment is a major concern for developed and developing countries alike. Governments around the world are constantly trying to control and reduce air pollution levels through regulations. Low emission zones are being designated in cities worldwide in order to reduce the level of pollutants in big cities. The automotive industry is affected by those regulations and they are becoming more demanding over the years. Present work is aimed at developing a control strategy for a hybrid vehicle in order to optimize the fuel economy and emission levels based on GPS information, driver specific driving characteristics and weather forecast data for a given route. It uses powertrain model of a hybrid vehicle for developing route and driver specific control strategy. The full vehicle model has two sub-models: a route selector and a powertrain optimization model. The route selector selects the optimum route for the vehicle based on energy consumption considering possible routes for reaching the destination. Then the performance is optimized for the selected route using a cost function, which considers route segments which include low emission zone and available data for the headwind during the journey. The results identified the option for choosing strategy for improving both fuel economy and emission levels for a given route.
\end{abstract}

\section{Introduction}

Air quality, especially, in urban areas presents a worldwide problem which affects all the countries alike. Since the overall quality of the air is highly affected by road transport, the European Union imposed the "Euro emissions standards" (EC-Environment 2016)I n order to reduce the pollutants emitted to the atmosphere. Emissions standards imposed by governments around the world are becoming more restrictive over the years. In 1992 the "Euro 1" standard was imposed, imposing the use of a catalytic converter to gasoline cars in order to reduce the amount of $\mathrm{CO}$ emissions produced. Over the years the regulations became more restrictive leading to a reduction of the emissions of some pollutants by $96 \%$ (AA 2015). In 2012, air pollution of cities or rural areas were estimated to cause 3.7 million premature deaths worldwide. The World Health Organization concluded in 2014 (WHO 2014) that the particulate matter component of air pollution is carcinogenic to humans with high incidence in lung cancer. Research linked to adverse health impacts also (Kampa and Castanas 2008) attribute symptoms such as nose and throat irritation followed by bronchoconstriction and dyspnoea to nitrogen oxides (NOx) and certain heavy metals to emissions from combustion devices. According to (European-Comission 2015), 310,000 premature deaths are attributed to air pollution each year. Road accidents cause less fatalities than air pollution. The economic impact of the human health damage caused by air pollution is estimated to be between $427 €$ and $790 €$ billion each year. With the evidence of the damage that pollution can cause to human health, countries around the world as well as the European Union are forced to tougher air quality standards. The imposed air quality standards are hard to achieve in big cities due to the high concentration of vehicles, Low Emission Zones (LEZ) were imposed in some of them to improve air quality. The impact of LEZs in European cities was positive on air quality terms. The city of Berlin reduced the NOx emissions of its whole fleet by $20 \%$ in just two years according to (EU 2015). The level of impact depends on different aspects such as the emissions standard set, the enforcement of the LEZ, the extension of it or the number of vehicles affected.

New regulations forced the automotive sector to find a solution for deteriorating ambient air quality and also replace the hydrocarbon based fuels with low carbon or carbon free fuels recently. This has resulted in hybridisation or electrification of the vehicles (Traders 2015).

Hybrid vehicles open the opportunity of drawing the energy needed to drive a car from different resources as batteries, internal combustion engines and flywheels etc. (Mohan, Assadian, and Longo 2014). The energy efficiency characteristics of those sources differ from one to another (Gross 2007, Heywood 1988) which makes energy management a crucial task. With the flexibility found in hybrid powertrains new control strategies can be developed in order to maximize the efficiency of the vehicle for specifics routes.

Conventional electric hybrid vehicles have reactive control strategies which are primarily governed by the state of charge of the battery and to sustain the SOC of the battery (Baumann et al. 2000) during the journey. This reactive strategy makes sure that the battery level is between the desired boundaries but it can be inefficient in some circumstances. Reactive control strategies are tuned based on standard driving cycles which mean that the vehicle performance can be close to the optimal in these circumstances but it does not guarantee the optimal operation over different scenarios (Zeng and Wang 2015). These control strategies can be improved by making use of the GPS data of the vehicle which can predict what the vehicle is

Page 1 of 9 
going to encounter in the road ahead and adapt its control strategy in order to maximise efficiency (Zou et al. 2013).

Hybrid powertrain energy management strategies have been an object of study for years and can be classified in three main categories: rule based control, static optimization methods and dynamic optimization approaches. Rule based control strategies are implemented in production cars due to its light computational requirements but as mentioned previously, they cannot guarantee optimal performance for different scenarios (Baumann et al. 2000). Static optimization methods are positioned in an intermediate position with medium computational requirements and closer to the optimal if compared with the rule based control (Kim et al. 1999). Dynamic programming approach is hardly implementable into road vehicles because of its high computational requirements. However, it can be used to set benchmarks from which other control strategies can be compared against (Chan-Chiao et al. 2003).

The present work makes use of the data available from GPS system of a car, drive cycle data available for the route from the data bank and prevailing wind conditions from weather forecast in order to optimize the powertrain performance for a given journey. The model will be split in two different models which were linked and share information. The first part is a route selector which will select which is the optimum route for reaching the destination based on energy consumption. The energy consumption calculation will include vehicle speed, road grade and relative wind speed from the vehicle. Low emission zones (LEZ) appearance during a route will be detected by the route selector and it will transfer that information to the main model too. Once the optimal route is selected the route information is transferred to a more detailed and complex vehicle model which will vary the energy management system of the vehicle in order to drive through the route in an optimal manner. The main model will make use of a cost function which will define the optimization objective. Since the route selector has already identified the appearance of a LEZ during the route, the cost function will vary in order to meet the LEZ requirements, modifying the powertrain control based on emissions reduction or optimum fuel economy.

\section{Baseline reactive control strategy}

This project will be based on vehicle simulations. Vehicle simulations has been performed using GT-Suite software. The 2004 Toyota Prius powertrain known as Toyota Hybrid System II (THS-II) was used as baseline in the simulation performed in this project. The THS-II powertrain was selected because there are several studies of it since it set the benchmark of hybrid vehicles in the past (Meisel 2011, Yamamoto 2010, Duoba, Ng, and Larsen 2001). The overall layout of the model can be seen in Figure 1.

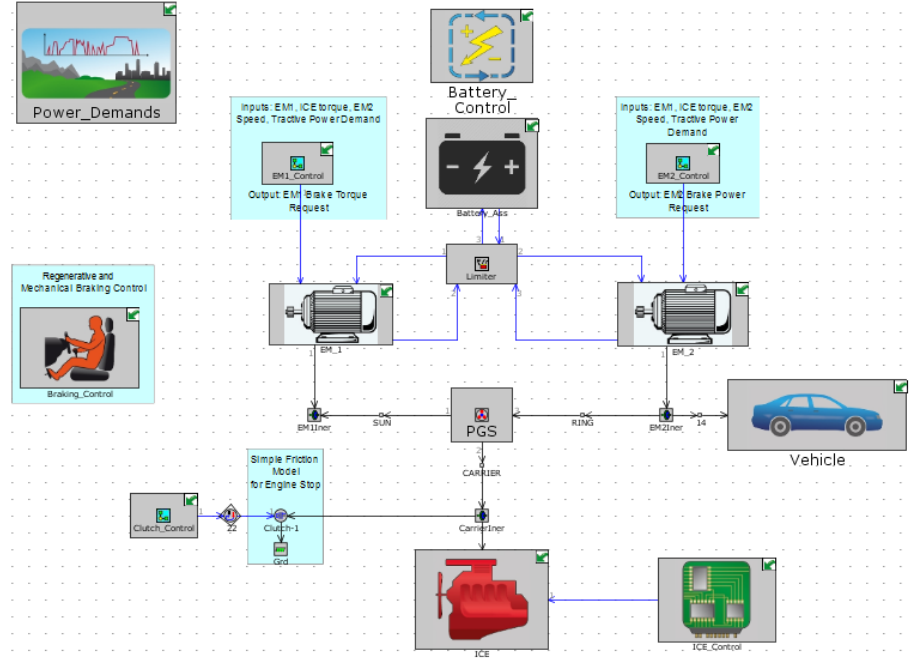

Figure 1: Toyota THS-II model layout (Gamma-Technologies 2015)

The vehicle module represents a physical model of the dynamic of a conventional vehicle which estimates the torque required to drive the vehicle for a given vehicle speed. The vehicle is connected to the road through axles and wheels which inertia can be specified in order to take them into account in the calculation. The road module contains information of road grade, road curvature radius and rolling resistance changes due to changes in road surface. The vehicle is also connected to the ambient were information of air density, air velocity and relative air direction with respect of the vehicle can be specified. The tractive power of the vehicle comes through the driveshaft which comes from the transmission to the front differential. The differential transmits the tractive power through the front wheels to the road.

The ICE object is a map-based model used to characterize the engine performance, fuel consumption, heat rejection and emissions. In this particular model, the engine had the accelerator opening percentage as an input and it returned engine torque and engine speed. Since we did not have detailed emission maps for THS-II, the emissions maps of a typical EURO-IV vehicle obtained from a controlled experiment with the similar loading conditions were used in this model. Although this approach will not represent the actual vehicle's emissions levels from THS-II, it will be sufficient for comparing the performance of a baseline vehicle with that of optimum control strategy.

The model estimates the power required to drive the vehicle at the speeds dictated by the speed profile imposed, this will be an estimation of the total power required to drive the vehicle based on the speed profile and vehicle mass and inertia characteristics. Another crucial component that has to be managed efficiently in a hybrid vehicle is the battery. For the baseline model there is a specific module to control this component. The objective of this control is to determine the power required by the battery in order to maintain the SOC between its boundaries. The SOC demand is converted into power demand necessary from the ICE in order to maintain the battery levels. Modification in this control could make the system more flexible when controlling the battery levels, requiring less power from the engine in some situations.

The THS-II features regenerative braking which is used to recharge the battery in deceleration periods. The vehicle can brake making use of the electric motors or by using conventional friction brakes. The generator has the objective of controlling the ICE speed and force it to run in its most efficient range whenever it is possible. This control 
has been specifically tuned to reduce fuel consumption without having the emissions into account. The last important component of the vehicle's layout is the electric motor, it has the objective of supplying additional tractive power when required and also regenerating energy when the regenerative braking is enabled.

\section{Main model validation}

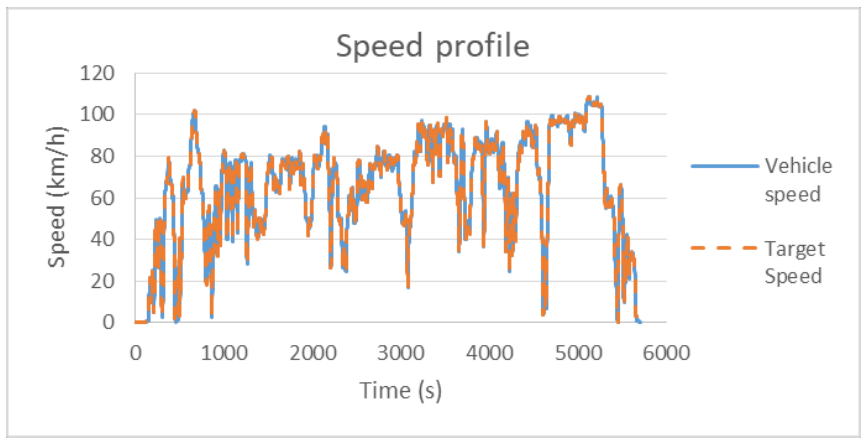

Figure 2: Comparison of target vehicle speed against actual vehicle speed achieved by the model

With the purpose of testing the accuracy of the model a comparison between the input speed profile which the vehicle will try to follow and the actual vehicle aped achieved by the model can be seen in Figure 2. As can be observed, the accuracy of the speed controller is more than acceptable with the vehicle following thoroughly the input curve.

In order to continue validating the model a comparison between experimental data and the results from the simulation was performed. The experimental data was extracted from (Duoba et al. 2001) where a Toyota Prius equipped with extra sensors was tested following the US06 driving cycle speed profile. The results for a section of the drive cycle were compared for the same section done in the simulation. The results are shown in Figures 3 and 4.

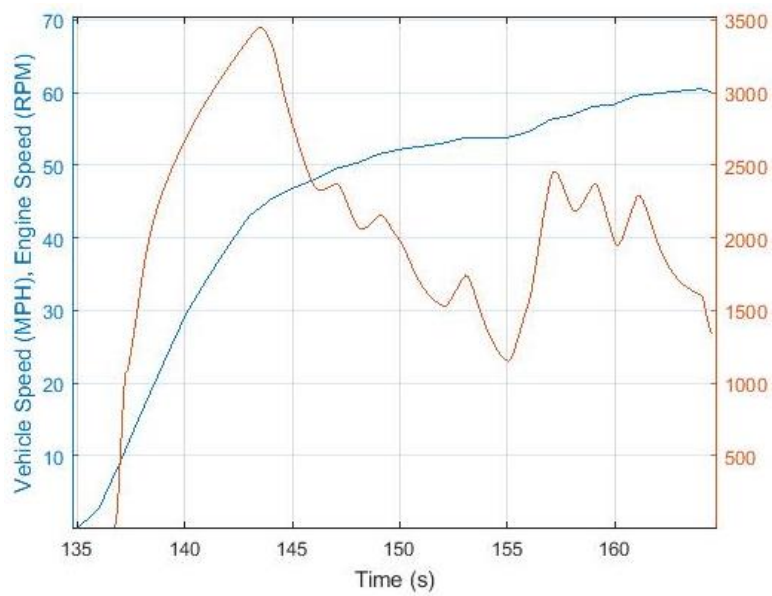

Figure 3: Engine Speed, in red, and vehicle speed, in blue, as a function of time for following US06 drive cycle from the powertrain model.

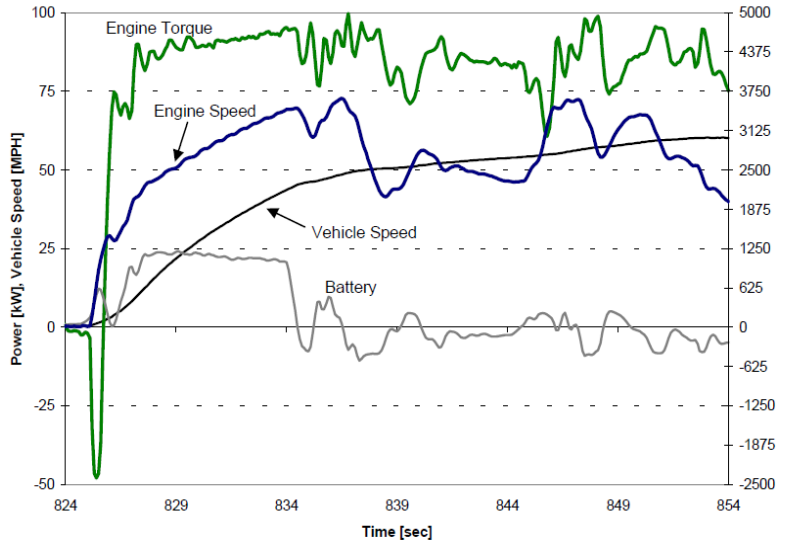

Figure 4: Operating conditions of the powertrain as a function time for the same portion of US06 drive cycle from published literature experimental data (Duoba, Ng, and Larsen 2001)

The vehicle speed profiles are identical which indicates that the power demand control is set up correctly. However, there are some clear differences when the engine speed is compared, with much higher engine speeds in the real test compared to the simulation model. The main reason why this is happening is because of the control of the model is forcing the engine to be at lower speeds to reduce fuel consumption. The engine is forced to follow be at lower speeds compared to what the experimental data from the vehicle shows. This control will help to reduce fuel consumption but it is ignoring its effect on the emissions produced by the ICE. Since the emissions are one important aspect considered in this project, the relaxation of the model in this area will be crucial to control the emissions levels of the vehicle.

A maximum acceleration test was performed in order to validate the maximum capabilities of the vehicle. From (Muta, Yamazaki, and Tokieda 2004) it was possible to extract the curve of maximum driving force for the THS-II powertrain as shown in Figure 5. If the graph is compared with the one obtained by simulating the model as shown in Figure 6, it is possible to see clear differences:

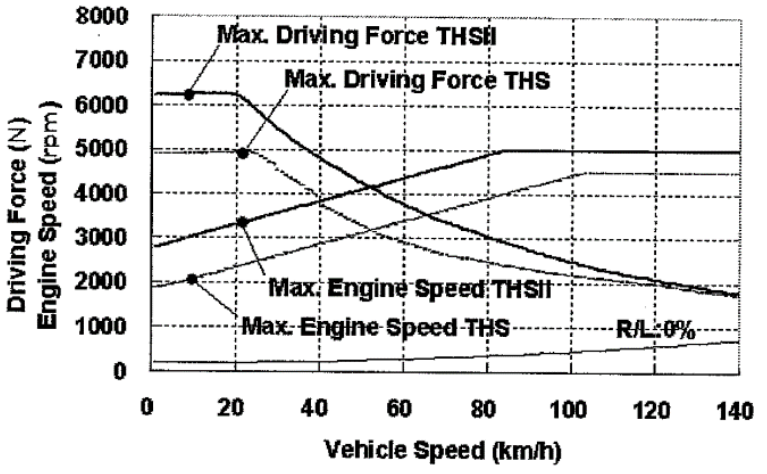

Figure 5: Maximum tractive force of THS and THS-II from (Muta, Yamazaki, and Tokieda 2004) 


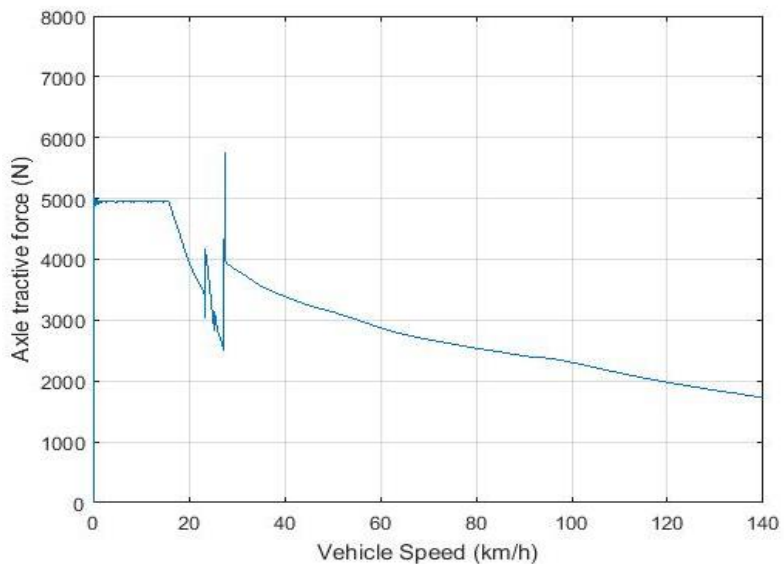

Figure 6: Maximum tractive force from present model

The tractive force has some irregularities from the seconds 20 to 25 and in overall terms the tractive force follows the tractive force of the THS powertrain instead of following the THS-II curve which is the powertrain which is supposed to be modelling. The reason of this difference is because the model engine control was optimised for minimise fuel consumption and its control is not the same as the one that can be found in the standard vehicle.

As a conclusion, the validation process showed that the baseline simulation model was clearly optimised to minimise fuel consumption. A NEDC simulation was run in order to compare the fuel consumption of the model with the results of the THS II powertrain from the Vehicle Certification Agency (VCA 2004). The result showed a significantly lower fuel consumption (21\%) predicted by the model. The fact that the baseline model was optimised for low fuel consumption will present the opportunity of showing how emissions are affected by the change in powertrain control.

\section{GPS Based Model}

The simulation model has been separated into two different models: a route selector and a main optimization model. The main objective of the first model is to select the route with less energy demand. The outputs from the route selector will be used as inputs for the main model which will run an optimization problem in order to minimise a pre-defined cost function as shown in Figures 7 and 8.

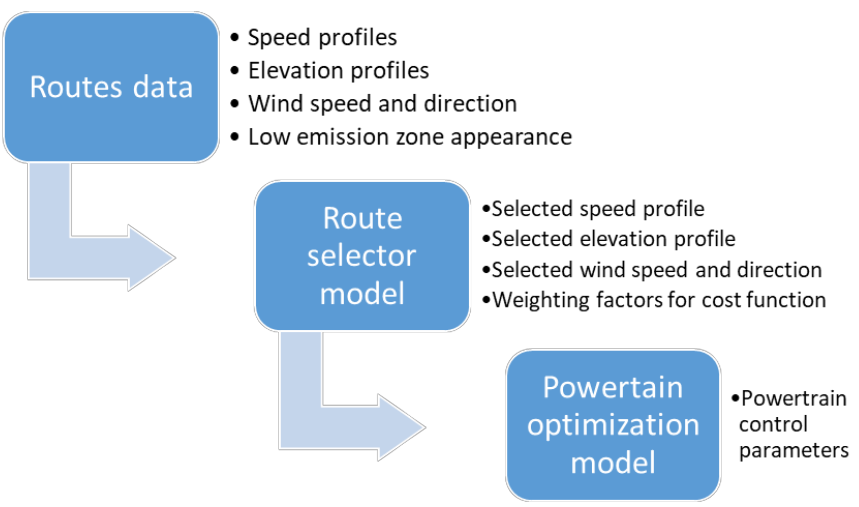

Figure 7: Scheme of work and flow of information

\section{Route Selector}

This model makes use of simple equations to determine the amount of energy needed by a standard vehicle to drive through different routes for the same destination and select the route which consumes the least amount of energy. Furthermore, it will select the weighting factors used by the cost function of the main model. In order to calculate the approximated energy consumption, the route selector model will need various inputs as speed profiles, elevation profiles, location points (latitude and longitude), local wind speed and local wind direction for each alternative route.

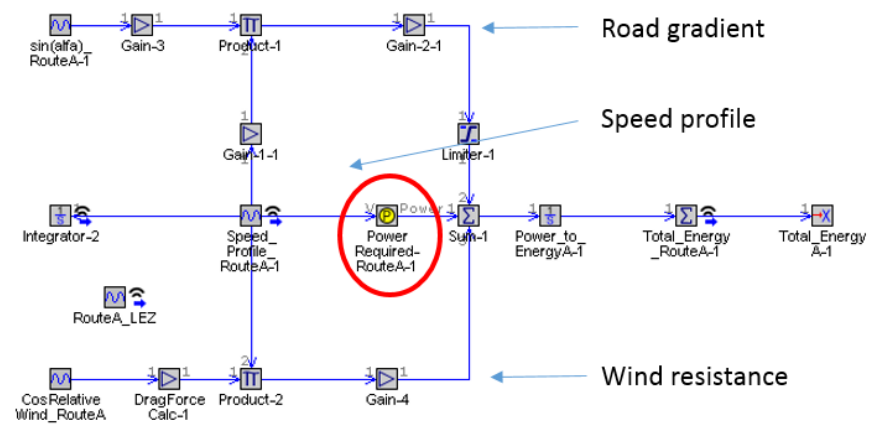

Figure 8: Scheme used for estimating energy required to follow the route

Figure 8 shows how the calculation of the predicted energy consumption for one of the routes is calculated and its general structure. There are three main branches which calculate the road gradient, the vehicle speed and the wind resistance power along the route. All these effects are added and then integrated in order to obtain the energy consumption. The controller module highlighted in Figure 8 is a predefined controller which will calculate the tractive power required in order to allow the vehicle to follow a given speed profile. The power demand calculated by this module accounts for tyre rolling resistance and road curvature effects. The power request also includes the necessary power to accelerate or decelerate the vehicle and cargo mass as well as major vehicles inertias as axle and wheel inertias and the effect of the driveline efficiency. The output of the previous controller will be added to the aerodynamic resistance power $\left(P_{A R}\right)$ and the road grade resistance power $\left(P_{R G}\right)$. The resistance force due to road gradient is given by Equation 1 .

$F_{R G}=m \cdot g \cdot \sin (\propto)$

Where $m$ is the mass of the vehicle, $g$ is the gravitational acceleration and $\alpha$ is the road inclination. Since the instantaneous power of a force applied to an object travelling at a given speed is calculated as:

$P=F \cdot v \cdot \cos (\theta)$

Where $P$ is the instantaneous power, $F$ is the force applied to a body, $v$ is the velocity of the force application point and $\theta$ is the angle between the force and velocity vectors. Since the resistance force due to gradient is considered to affect only the longitudinal direction of the vehicle, the cosines term can be suppressed. If equations 1 and 2 are combined the resistance power due to road gradient will be:

$P_{R G}=m \cdot g \cdot \sin (\propto) \cdot v$

Page 4 of 9 
In order to calculate the energy consumption due to wind resistance the relative wind speed and direction with respect to the vehicle is required. Making use of the latitude and longitude data available from the GPS data and the wind speed and direction on that region from the weather forecast data at that time a MATLAB script (MathWorks 2015) was developed in order to calculate the relative wind speed and direction with respect of the vehicle. It is important to state here that the wind speed and direction was supposed to be constant for the whole simulation as the real time update of the wind maps was not achievable with GT-Drive software. In order to calculate the relative wind direction, the route location points and the local wind direction are used. Vector of displacement between location points are calculated and expressed in polar coordinates. The angle of the vectors are subtracted from the local wind direction in order to obtain the relative wind direction. The aerodynamic resistance force calculation is given by equation 4 .

$F=\frac{1}{2} \rho C_{d} A v^{2}$

Where $\rho$ is the air density, $C d$ is the drag coefficient, A is the frontal area of the vehicle and $\mathrm{v}$ is the relative velocity between the vehicle and the air. Since the most significant changes in energy consumption due to drag are in the longitudinal direction of the vehicle it is important to calculate the aerodynamic force in the longitudinal direction, this was done incorporating a cosine term with the angle formed between the vehicle and the air. Making use of Equation 4 the calculation of the aerodynamic resistant power becomes:

$F_{A R}=\frac{1}{2} \rho C_{d} A \cos (\theta) v^{3}$

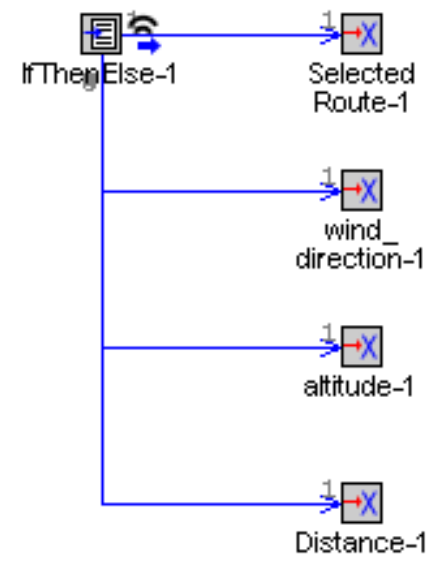

Figure 9: List of output signals from the route selection model

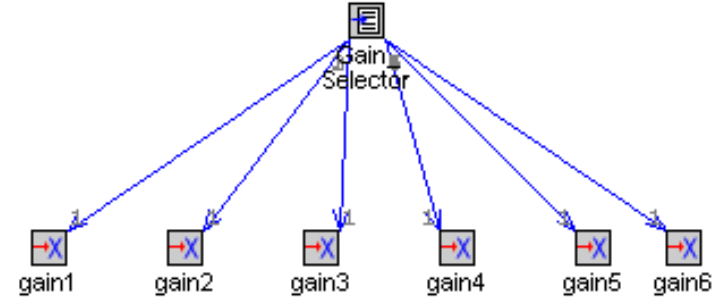

Figure 10: Scheme used for choosing appropriate weights for individual signals for the cost function

Figure 9 selects the speed profiles, altitude profiles and wind direction profiles based on the total energy consumption calculated by the previous section. Figure 10 selects the gain values of the cost function of the main model. This will change the optimization's objective based on the appearance of a LEZ during a route. The gains used in the simulations and they origin will be explained in the following section. Once the calculation of the energy needed for each route is done the model selects the optimal route and generate different outputs to be used by the main model:

- Speed profile of selected route

- Road elevation profile of the selected route

- Relative wind direction of the selected route

- Weighting factors for the cost function included in the main model

\section{Powertrain Optimization Model}

The main goal of the optimization problem is to minimize a cost function for different outputs of the vehicle. Different optimization studies for hybrid powertrains have been done in the past and they used just one parameter to optimise different aspects from the vehicle by the implementation of a cost function. Different cost functions have been used for different purposes; Chan-Chiao et at (Chan-Chiao et al. 2003) used a cost function that took into account emissions and fuel consumption of a heavy duty vehicle while Yan et al (Yan, Wang, and Huang 2012) used a cost function that took fuel consumption, battery depletion and number of engine starts and stops into account. The cost function employed in this present work is shown in equation 6 .

$J=\int_{t i}^{t f}\left(\alpha_{1} * P_{B}(t)+\alpha_{2} * f \dot{u e l}(t)+\alpha_{3} * N \dot{O} x(t)+\alpha_{4} * \dot{H} C(t)+\right.$
$\left.\alpha_{5} * \dot{C O O}(t)+\alpha_{6} * \dot{C} \dot{O}_{2}(t)\right) d t$

Where $P_{B}$ is the maximum available power from the battery, this term controls the battery depletion since the maximum available power depends on the state of charge of the battery. The rest of the terms are the consumption rate of fuel $(f u e l(t))$ and the emissions rate of $N O x$, $\mathrm{HC}, \mathrm{CO}$ and $\mathrm{CO} 2$ respectively. All the terms that appear in the cost function have an associated weighting factor $(\alpha i)$.

The values of emissions, fuel consumption and available power in the battery were normalised in order to make the different measures comparable. The weighting factors $(\alpha i)$ are used to modify the weight of each parameter according to what the user finds appropriate in a specific case. In the simulations performed during this project the weighting factors were selected depending the appearance of a low 
emission zone during the route. If the route selector detected a low emissions zone during the journey, more weight will be given to $\mathrm{NOx}$ emissions than fuel consumption.

\section{Relaxation of the model}

In order to allow the optimization process, the main two components that need to be controlled are the two power sources, in this case the ICE and the battery. The modification of the ICE control was done through the modification of the generator control which was responsible of the control of the ICE speed. The baseline curve followed by the model was fixed forcing the engine to run at the given speeds depending on the demanded power. The new curve features two sliding points which allow the curve to modify its shape, allowing the engine to be run at different speeds depending on the conditions imposed by the cost function. Battery control modification was made by the introduction of a new variable in the battery SOC control. The target SOC of the battery was set as new variable allowing the battery to be more flexible when demanding power to the internal combustion engine.

\section{Simulation of GPS based model}

In order to perform realistic simulations data from known trips were used in the simulation. The route data was logged making use of the GPS module and it represents two alternative routes between the cities of Oxford and Coventry as shown in Figure 11.

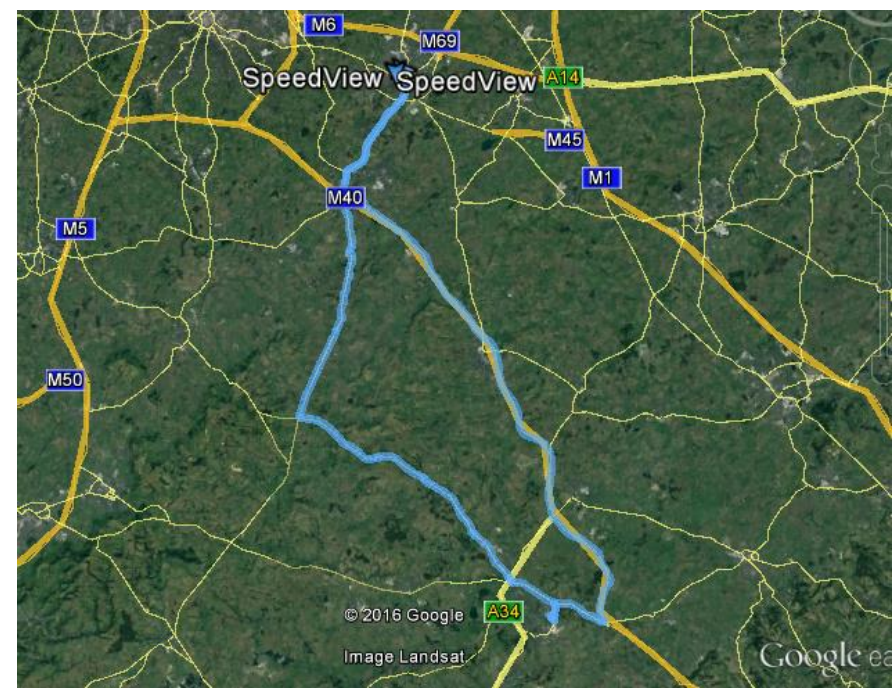

Figure 11: Alternative routes chosen for same the destination for developing route specific vehicle speed profile

The selected routes consist of a high speed motorway trip $(106.5 \mathrm{~km})$ and a lower speed route $(104.4 \mathrm{~km})$ which goes through different towns and cities. Different driving patterns are observed in the speed profiles of the routes as can be seen in Figure 12.

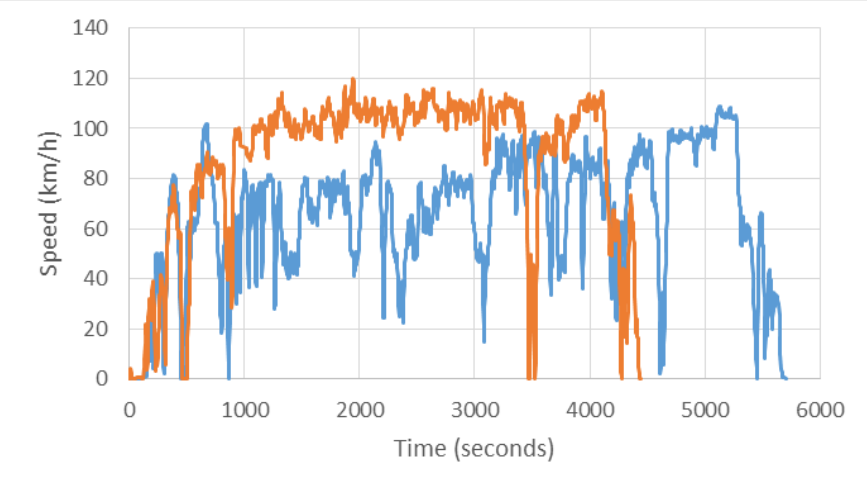

Figure 12: Vehicle Speed profiles for alternative routes

The resulting speed profiles after the filtering process are shown in Figure 12. The motorway trip has much higher vehicle speeds and a much more constant speed profile if compared to the no-motorway trip. The trip that followed mainly A-roads has lower and more inconsistent speed values in comparison.

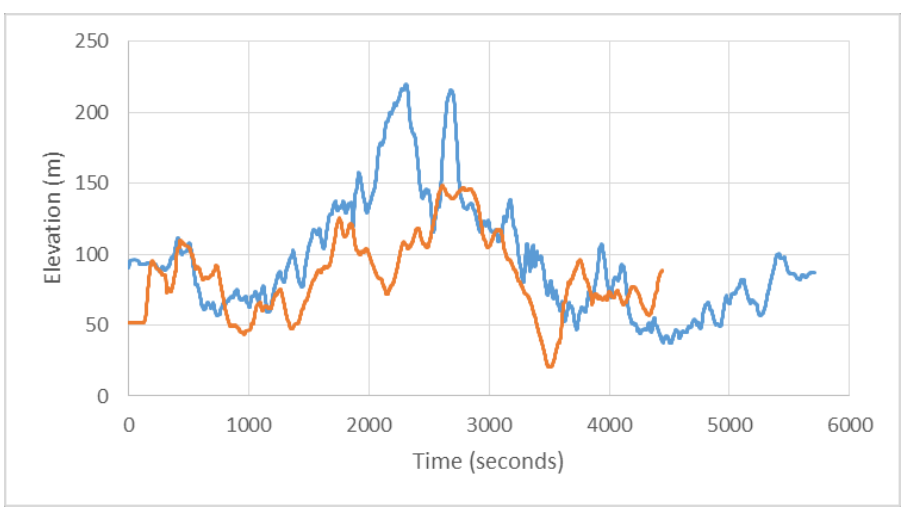

Figure 13: Road elevation profiles of alternative routes

The elevation profiles of both routes can be seen in Figure 13, the motorway trip shows a much smoother elevation profile if compared to the conventional road route. The elevation profiles were available from the GPS data recorded. The wind direction and speed for both routes were different since the routes were recorded on different days. The data for this study was obtained from MetOffice (MetOffice 2016).

\section{Results and discussion}

In order to assess the effect of the route selector the two alternative routes are compared in terms of energy consumption and emissions levels. The fuel consumption of the A-roads trip was $11.3 \%$ less than what the motorway trip registered. The main difference between both routes is the energy loss due to the aerodynamic drag with much higher losses for the motorway trip. Once the route is selected the powertrain of the vehicle will be optimized to run efficiently through it. Since the powertrain control will change if a LEZ is detected through the route, two different cases were studied. A first one with no appearance of LEZ in which the main objective would be to minimise fuel consumption and a second one which will include a LEZ and the main objective will be to reduce the emission levels. The results show an improvement in fuel consumption and emission level over the baseline model in both scenarios. In the case of 
minimum engine fuel consumption, the improvement over the baseline is of $0.72 \%$ while in the case of minimum NOx emissions the fuel consumption is reduced by $0.63 \%$. The fuel consumption is very close to the results achieved by the baseline model, this is due to the fact that the baseline model was specifically designed for minimum fuel consumption as explained in previous sections. In order to put the results in context it is important to remember that the baseline model consumed $21 \%$ less fuel if compared to the real vehicle (VCA 2004).

When the model was optimized for better fuel economy, the NOx emission levels decreased by $2.3 \%$ from that of baseline, but in contrast, when the vehicle was optimized for emission levels, the NOx emissions were reduced by $5.3 \%$ when compared to baseline. It is important to highlight that the fuel consumption and NOx emissions cannot be reduced at the same time since what is good for reducing fuel consumption might not be good for reducing NOx emissions. This difference is due to the fact that the production of NOx happens mostly at low engine speeds in this engine while the minimum fuel consumption is generally benefitted by low engine speeds. This situation leads to a trade-off between NOx generation and fuel consumption which can be controlled and modified adjusting the weighting factors of the cost function.

In order to fully assess the vehicle performance through the route not only the fuel consumption or the emissions have to be considered. Since the vehicle tested is a hybrid vehicle with a battery which can store energy, one important aspect to look at is the added or depleted energy of the battery. If during one route more battery energy is added to the battery, then that energy is available to be used by the vehicle for the next trip. To compare the results of the simulations the energy stored in the battery was converted to its fuel-equivalent. The following equations were used in order to convert the extra energy stored in the battery into equivalent mass of fuel.

$m_{\text {efuel }}=\Delta E_{\text {elec }} k . L H V$

$k=\frac{\eta_{I C E} \eta_{\text {trans }}}{\eta_{\text {batt }} \eta_{e m}}$

Where $m_{\text {efuel }}$ is the equivalent mass of fuel saved, $\eta_{I C E}$ is the thermal efficiency of the engine, $\eta_{\text {trans }}$ is the transmission efficiency, $\eta_{b a t t}$ is the battery efficiency, $\eta_{\mathrm{em}}$ is the efficiency of the electric motors, $L H V$ is the low heating value of the fuel and $\Delta E_{\text {elec }}$ is the difference between the energy stored in the battery between the optimized models and the baseline.

\section{Fuel consumption improvement}

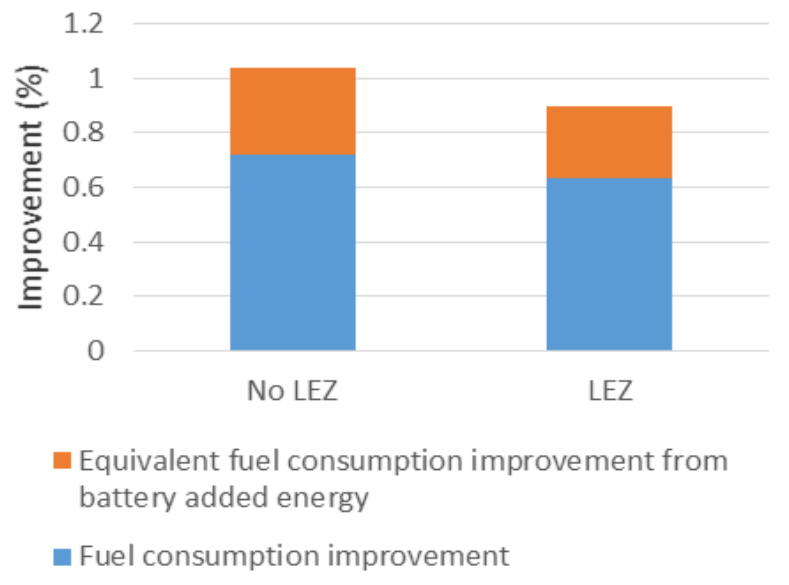

Figure 34: Estimated improvement for Fuel consumption value

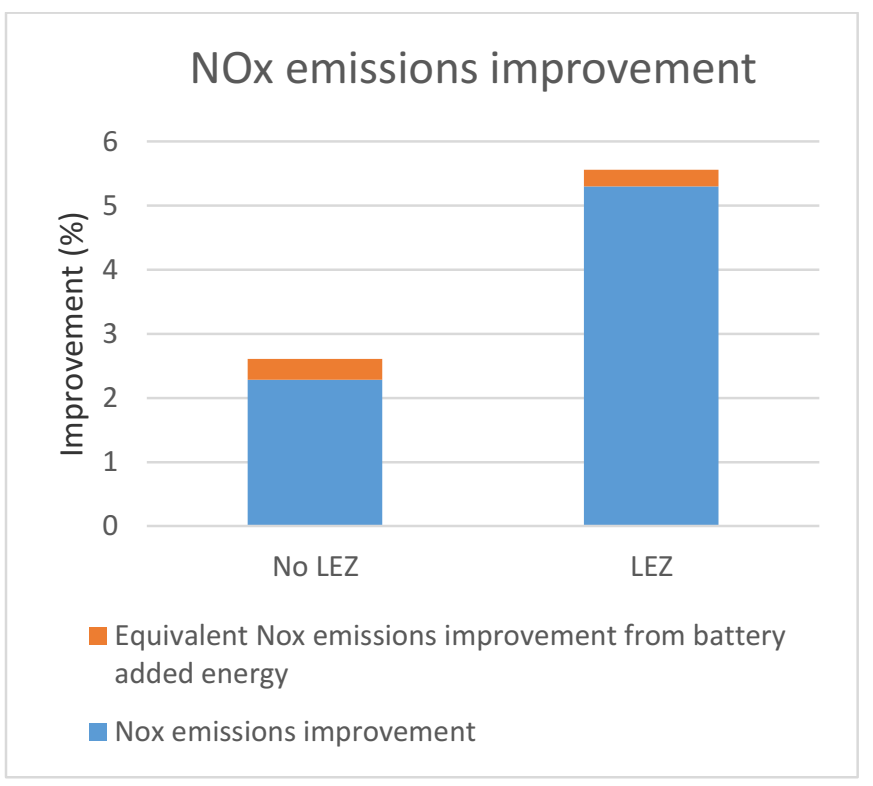

Figure 45: Estimated improvement for NOx emission levels

The added energy to the battery will not only affect fuel consumption but emissions too. If the fuel consumption savings are extrapolated to emissions savings the equivalent NOx emissions saving can be calculated as follows.

$N O x_{e q}=\frac{m_{e f u e l} N O x_{\text {total }}}{m_{t f u e l}}$

Where $m_{e f u e l}$ is the equivalent mass of fuel saved due to the added energy to the battery, $m_{t f u e l}$ is the total mass of fuel consumed during one trip and NOx total is the total mass on NOx emitted during the trip.

Figures 14 and 15 show the effect of any difference between the SOC of the battery between the different simulations. The blue portions of the figure show how much the fuel consumption and the NOx 
changed between the baseline control and the new powertrain control. In contrast, the orange portions of the figures show what the improvement was once the difference in SOC of the battery is taken into consideration.

The results shown in Figures 14 and 15 indicate that the equivalent energy stored in the battery has a higher effect in the fuel consumption than in the NOx emissions in comparison with the savings already achieved. The reason of it remains on the fact that the fuel consumption savings were not as great as the emissions saving so, in comparison, the added energy to the battery will have a higher effect on the fuel consumption.

\section{Overall model results}

In the previous sections the performance of main features of the model was assessed, in this section the overall performance of the optimization process are being compared. The total fuel consumption improvement of the vehicle is calculated as the addition of the fuel economy gain due to the route selector and the fuel economy gain due to the powertrain optimization. The savings on emissions are of more than $5 \%$ in the case of the appearance of a LEZ which is when the powertrain is mostly optimized to NOx emissions reduction.

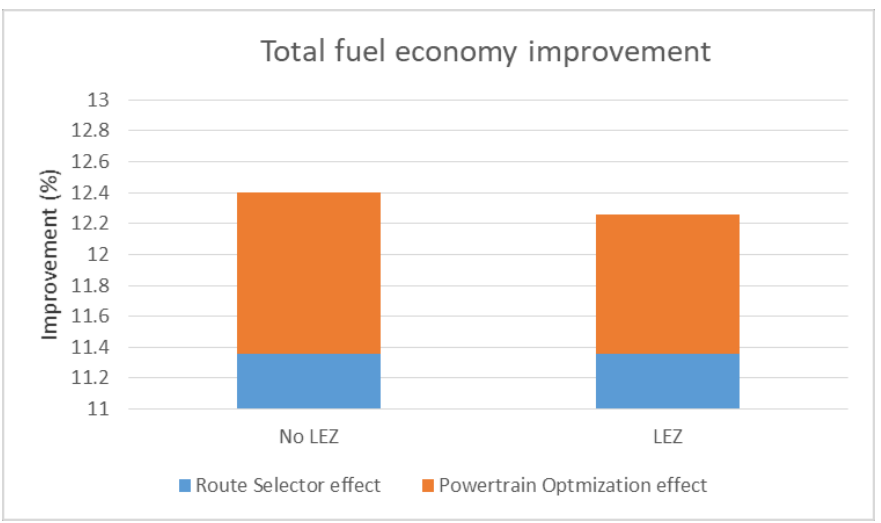

Figure 56: Estimated improvement for overall fuel economy level between baseline model and the new control proposed

As can be seen in Figure 16, the major gain in terms of fuel consumption are due to the effect of the route selector which reduced $11.3 \%$ the fuel consumed by the vehicle when travelling between the start and the finish point. Furthermore, the powertrain optimization adds an additional $1 \%$ and $0.9 \%$ fuel efficiency gain for the cases the route without a LEZ and the route with a LEZ respectively. The overall result shows an improvement of over $12 \%$ in fuel efficiency for both cases. Overall, an improvement on fuel economy and emissions can be observed in both cases. There is a trade-off between fuel economy and NOx emissions in the vehicle and the cost function defines the point where the vehicle will operate.

\section{Conclusions}

Previous work in the literature presented different approaches for optimizing a hybrid vehicle powertrain. Mainly they used GPS data in order to optimize the fuel economy and emissions performance of a vehicle through a given route. Dynamic optimization showed a positive effect when applied to powertrain optimization problems. However, there was the lack of a process that combine route selection with powertrain optimization.
The implementation and growth of low emission zones over the recent years opened a good opportunity to highlight the importance of the linkage of the route selection with the powertrain optimization.

The developed model makes use of the data available in the GPS system of a car in order to optimize a journey to a destination. The model is split in two different models which were linked and share information. The first part is a route selector selects which is the optimum route for the vehicle to follow based on energy consumption through different routes. The energy consumption calculation during the routes includes vehicle speed, road gradient and relative wind speed from the vehicle. LEZs are detected and the weighting factors of the cost functions are set according to it. Once the optimal route is selected the route information is transferred to a more detailed and complex vehicle model which varies the energy management system of the vehicle in order to drive through the route in an optimal manner. The main model uses of a cost function which will define the optimization objective. Since the route selector has already identified the appearance of a LEZ during the route, the cost function will vary in order to meet the LEZ requirements, modifying the powertrain control based on emissions reduction or optimum fuel economy.

The cost function presents a very simple and effective way of adapting the control strategy to vehicles which have to meet different requirements given by legislation changes or just to meet certain manufacturer's standards. It also opens the opportunity to adapt the control strategy to vehicles which are not even hybrid which can benefit from both route selection and powertrain optimization as well. In order to adapt it just changes in the optimization variables will have to be made. This fact highlight one of the main strengths of the presented control strategy, its versatility.

The results from the performed simulations showed an improvement in fuel economy and emissions for all the presented cases. The route selector helped to improve fuel economy by $11 \%$ just selecting the less energy consuming route. In addition to it, the powertrain optimization model reduced fuel consumption by an additional $1 \%$. The NOx emissions which were set as main emission target were reduced by $2.5 \%$ in the case of a route which does not include a LEZ in it and by more than 5\% in the case of the appearance of a LEZ.

Route selection plays a very important role in the optimization process. Furthermore, the versatility of the cost function allowed the vehicle to adapt its optimization target depending on the demands encountered in the route.

\section{References}

AA. Euro emissions standards 2015 [cited 28/08/2016. Available from https://www.theaa.com/motoring advice/fuels-andenvironment/euro-emissions-standards.html.

Baumann, B. M., G. Washington, B. C. Glenn, and G. Rizzoni. 2000. "Mechatronic design and control of hybrid electric vehicles." IEEE/ASME Transactions on Mechatronics no. 5 (1):58-72. doi: 10.1109/3516.828590.

Chan-Chiao, Lin, Peng Huei, J. W. Grizzle, and Kang Jun-Mo. 2003. "Power management strategy for a parallel hybrid electric truck." IEEE Transactions on Control Systems Technology no. 11 (6):839-849. doi: 10.1109/TCST.2003.815606.

Duoba, Michael, Henry Ng, and Robert Larsen. 2001. Characterization and Comparison of Two Hybrid Electric Vehicles (HEVs) - Honda Insight and Toyota Prius. SAE International.

EC-Environment. Transport emissions, air pollutants from road transport. European Comission 2016 [cited 28/08/2016.

Page 8 of 9 
Available from

http://ec.europa.eu/environment/air/transport/road.htm.

EU. Impact of Low Emission Zones 2015 [cited 28/08/2016.

Available from http://urbanaccessregulations.eu/lowemission-zones-main/impact-of-low-emission-zones.

European-Comission. Urban Access Regulation In Europe 2015 [cited 28/08/2016. Available from http://urbanaccessregulations.eu/low-emission-zonesmain/what-are-low-emission-zones.

Gamma-Technologies. GT-Suite software 2015 [cited 28/08/2016. Available from https://www.gtisoft.com/.

Gross, Charles A. 2007. Electric machines. Boca Raton, FL: CRC Press.

Heywood, J. 1988. Internal Combustion Engine Fundamentals: McGraw-Hill Education.

Kampa, Marilena, and Elias Castanas. 2008. "Human health effects of air pollution." Environmental Pollution no. 151 (2):362367. doi: http://dx.doi.org/10.1016/j.envpol.2007.06.012.

Kim, Chunho, Eok NamGoong, Seongchul Lee, Talchol Kim, and Hyunsoo Kim. 1999. Fuel Economy Optimization for Parallel Hybrid Vehicles with CVT. SAE International.

MATLAB R2015a. The Mathworks, Inc.

Meisel, Jerome. 2011. "Kinematic Study of the GM Front-Wheel Drive Two-Mode Transmission and the Toyota Hybrid System THS-II Transmission." doi: 10.4271/2011-01-0876.

MetOffice. Weather Observation Website 2016 [cited 27/08/2016. Available from http://wow.metoffice.gov.uk/.

Mohan, Ganesh, Francis Assadian, and Stefano Longo. 2014. Comparative Analysis of Multiple Powertrain Architectures based on a Novel Optimization Framework. SAE International.

Muta, Koichiro, Makoto Yamazaki, and Junji Tokieda. 2004. Development of New-Generation Hybrid System THS II Drastic Improvement of Power Performance and Fuel Economy. SAE International.

Traders, The Society of Motor Manufacturers and. 2015. SMMT New car registrations January 2016. SMMT.

VCA. Vehicle Certification Agency - Vehicles data may 20042004 [cited 22/06/2016. Available from http://www.dft.gov.uk/vca/.

WHO. Ambient (outdoor) air quality and health 2014 [cited 28/08/2016. Available from http://www.who.int/mediacentre/factsheets/fs313/en/.

Yamamoto, Masaya. 2010. Development of a Toyota Plug-in Hybrid Vehicle. SAE International.

Yan, F., J. Wang, and K. Huang. 2012. "Hybrid Electric Vehicle Model Predictive Control Torque-Split Strategy Incorporating Engine Transient Characteristics." IEEE Transactions on Vehicular Technology no. 61 (6):24582467. doi: 10.1109/TVT.2012.2197767.

Zeng, X., and J. Wang. 2015. "A Parallel Hybrid Electric Vehicle Energy Management Strategy Using Stochastic Model Predictive Control With Road Grade Preview." IEEE Transactions on Control Systems Technology no. 23 (6):2416-2423. doi: 10.1109/TCST.2015.2409235.

Zou, Yuan, Hou Shi-jie, Li Dong-ge, Gao Wei, and Xiao-song Hu. 2013. "Optimal Energy Control Strategy Design for a Hybrid Electric Vehicle." Discrete Dynamics in Nature and Society no. 2013:8. doi: 10.1155/2013/132064. 